\title{
Spontaneous Necrosis of Choroidal Melanoma
}

\author{
Shalini Thareja ${ }^{a} \quad$ Alia Rashid $^{a}$ Hans E. Grossniklaus ${ }^{a}$ b \\ Departments of a Ophthalmology and ${ }^{b}$ Pathology, Emory University School of Medicine, \\ Atlanta, Ga., USA
}

\section{Key Words}

Necrosis · Choroidal melanoma $\cdot$ Uvea $\cdot$ Watershed infarction

\begin{abstract}
Background/Aims: The purpose of this study was to examine the clinical presentations and pathological features of spontaneously necrotic choroidal melanomas. Methods: The clinical and histological features of patients who underwent enucleation for uveal melanoma from 1989 to 2012 at Emory University and were found to have spontaneously necrotic choroidal melanomas were retrospectively analyzed. Results: A total of 6 cases were identified. All cases had $90-100 \%$ tumor necrosis and also exhibited marked ischemic necrosis of the iris and ciliary body; 5 of 6 cases exhibited marked ischemic necrosis of the retina. The tumor consisted of melanoma ghost cells often surrounded by a zone of pigmented macrophages. Thrombi were not found in any of the cases. All of the tumors in our cases were centered around the equatorial choroid and 2 extended into the ciliary body. One of the cases exhibited a wedge-shaped infarct in a lateral aspect of the tumor. In most of the cases, microscopic areas of intact tumor cells were present in the peripheries of the tumors. Conclusions: Spontaneous necrosis may occur in uveal melanoma. We believe that this occurs secondary to tumor hypoxia in the center of the tumor, followed by secondary inflammation, generalized ischemia and finally complete tumor necrosis.

(C) 2014 S. Karger AG, Basel
\end{abstract}

\section{Introduction}

Uveal melanomas can present on the iris, ciliary body, or choroid. Even in the presence of an extraocular extension, patients usually do not complain of pain unless there is ischemic necrosis in the tumor [1]. Previous studies have shown a range of presentations associated with necrotic uveal melanomas. In a study of 474 malignant melanomas, patients with necrotic 
Table 1. Clinical and pathologic characteristics of spontaneously necrotic choroidal melanoma

\begin{tabular}{|c|c|c|c|c|c|c|c|c|}
\hline Case & Sex & Age & Eye & $\begin{array}{l}\text { Tumor } \\
\text { extension }\end{array}$ & $\begin{array}{l}\text { Degree of } \\
\text { tumor necrosis }\end{array}$ & $\begin{array}{l}\text { AJCC } \\
\text { stage }\end{array}$ & $\begin{array}{l}\text { Ischemic necrosis } \\
\text { of ocular structures }\end{array}$ & Clinical symptoms and signs \\
\hline 1 & M & 65 & OS & $\begin{array}{l}\text { minimal invasion } \\
\text { of the sclera }\end{array}$ & $90 \%$ & pT4 & $\begin{array}{l}\text { iris, ciliary body, } \\
\text { retina }\end{array}$ & $\begin{array}{l}\text { blind painful eye, glaucoma, scleritis, } \\
\text { episcleritis, optic nerve gliosis, } \\
\text { retinal detachment, vitreous } \\
\text { hemorrhage }\end{array}$ \\
\hline 2 & M & 39 & OD & $\begin{array}{l}\text { through Bruch's } \\
\text { membrane to the } \\
\text { outer retina }\end{array}$ & $100 \%$ & $\mathrm{pT} 2 \mathrm{a}$ & $\begin{array}{l}\text { iris, ciliary body, } \\
\text { mildly ischemic } \\
\text { retina }\end{array}$ & $\begin{array}{l}\text { blind painful eye, glaucoma, scleritis, } \\
\text { optic nerve invasion anterior to } \\
\text { lamina cribrosa, vitreous } \\
\text { hemorrhage }\end{array}$ \\
\hline 3 & $\mathrm{~F}$ & 91 & OS & $\begin{array}{l}\text { superior temporal } \\
\text { vortex vein }\end{array}$ & $100 \%$ & pT3a & $\begin{array}{l}\text { iris, ciliary body, } \\
\text { retina }\end{array}$ & $\begin{array}{l}\text { blind painful eye, glaucoma, scleritis, } \\
\text { retinal detachment, vitreous } \\
\text { hemorrhage }\end{array}$ \\
\hline 4 & $\mathrm{~F}$ & 37 & OD & $\begin{array}{l}\text { invasion of the } \\
\text { sclera }\end{array}$ & $>90 \%$ & pT4b & $\begin{array}{l}\text { iris, ciliary body, } \\
\text { retina }\end{array}$ & $\begin{array}{l}\text { pain, scleritis, episcleritis, glaucoma, } \\
\text { retinal detachment, vitreous } \\
\text { hemorrhage }\end{array}$ \\
\hline 5 & M & 55 & OS & $\begin{array}{l}\text { through Bruch's } \\
\text { membrane }\end{array}$ & $>90 \%$ & pT3 & $\begin{array}{l}\text { iris, ciliary body, } \\
\text { retina }\end{array}$ & $\begin{array}{l}\text { NLP vision, left-sided headaches, } \\
\text { pain, scleritis, episcleritis, retinal } \\
\text { detachment, vitreous hemorrhage }\end{array}$ \\
\hline 6 & M & 31 & OD & $\begin{array}{l}\text { optic nerve, } \\
\text { conjunctiva, } \\
\text { invasion of the } \\
\text { sclera }\end{array}$ & $100 \%$ & pT4c & $\begin{array}{l}\text { iris, ciliary body, } \\
\text { retina }\end{array}$ & $\begin{array}{l}\text { blind painful eye, glaucoma, scleritis, } \\
\text { episcleritis, optic nerve invasion, } \\
\text { retinal detachment, vitreous } \\
\text { hemorrhage }\end{array}$ \\
\hline
\end{tabular}

OD = Right eye; OS = left eye, NLP = no light perception; AJCC = American Joint Commission on Cancer Staging Manual, ed 7.

tumors were more likely to present with glaucoma, inflammatory exophthalmos, rubeosis iridis, secondary angle-closure glaucoma, and inflammation of the uvea, sclera, and episclera compared to those without tumor necrosis [2]. Another study of 334 ciliary body and choroidal tumors showed that patients with necrotic tumors were more likely to present with episcleritis or scleritis than those without tumor necrosis [3]. Other studies have shown that patients with necrotic uveal melanomas may present with conjunctivitis, orbital inflammation [4], pain, and hyphema [5]. Individual case studies of spontaneously necrotic uveal melanomas have reported periorbital swelling and inflammation [6], vitreous hemorrhage, raised intraocular pressure [5], and occasionally uveal effusion [1], ophthalmoplegia, and proptosis [6]. In order to further understand the pathogenesis of spontaneous necrosis in uveal melanomas, we retrospectively reviewed patients who underwent enucleation for uveal melanomas and were found to have spontaneous tumor necrosis.

\section{Methods}

After obtaining approval from the Emory University's Institutional Review Board, we retrospectively reviewed all cases of enucleated eyes accessioned in the L.F. Montgomery Ophthalmic Pathology Laboratory between 1989 and 2012 that exhibited spontaneous tumor necrosis. Exclusion criteria included any treatment of the tumor prior to enucleation and necrosis occupying $<90 \%$ of the tumor volume. The patients' eyes were fixed in $4 \%$ formaldehyde and routinely processed, and 10 sections through the center of the pupil, optic nerve and melanoma were stained with HE and periodic acid-Schiff. A total of 6 cases were identified and analyzed for age, sex, left/right eye, clinical presentation, location of tumor, degree of necrosis, pattern of necrosis, and associated histological findings. 
Ocular Oncology

and Pathology
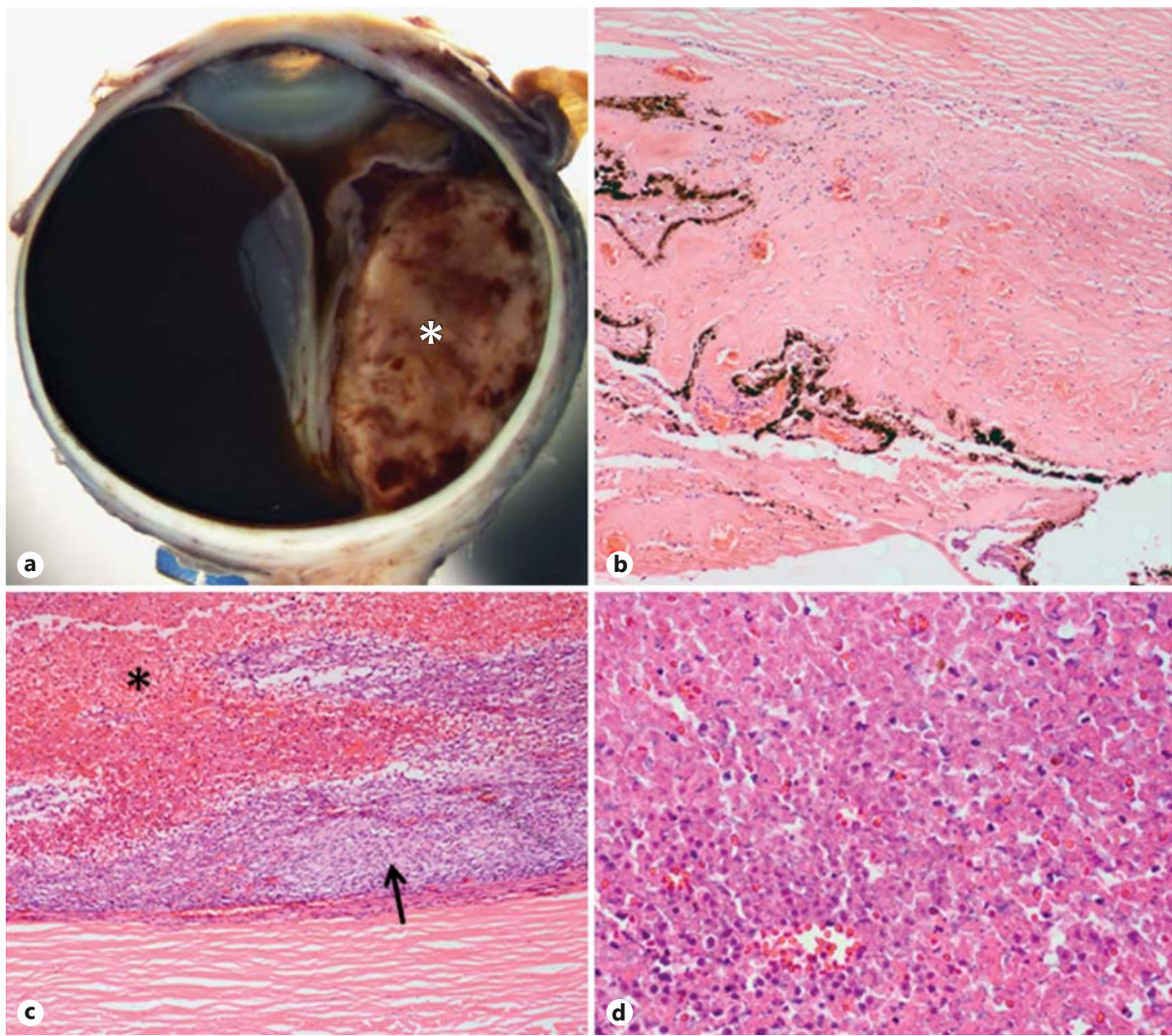

Fig. 1. Case 1. a Gross appearance of the enucleated eye with a dome-shaped melanoma (*) straddling the equator and an associated complete retinal detachment. b Ischemic necrosis of the ciliary body demonstrated by smudgy, pale staining and clumping of the iris pigment epithelium. c $90 \%$ of the tumor exhibits ischemic necrosis with associated hemorrhage $(*)$, and there is a microscopic rim of the intact tumor (arrow) at the periphery of the melanoma near the sclera. $\mathbf{d}$ The necrotic tumor consists of melanoma ghost cells with pale-staining cytoplasm and nuclei that occasionally exhibit basophilia. b, c HE. $\times 25$. d HE. $\times 100$.

\section{Results}

Our results are summarized in table 1 . Our patients had a mean age of 53 years, and there were 2 female and 4 male patients. Three melanomas were located in the left eye and 3 in the right eye. All patients in our study presented with pain, vitreous hemorrhage, overlying retinal detachment, and poor vision. All 6 patients had raised intraocular pressures up to including $60 \mathrm{~mm} \mathrm{Hg}$ and 5 suffered from sudden blindness in the involved eye. On examination, all 6 patients had what was thought to be scleritis, 5 exhibited what was interpreted to be episcleritis, and 4 had evidence of glaucomatous damage to the retina. One case exhibited extraocular extension to the conjunctiva and another case exhibited vortex vein invasion.

Representative pathological findings in cases 1 and 2 are show in figures 1 and 2, respectively. In all cases, tumor necrosis was $\geq 90 \%$, and the necrotic tissue was often associated 
Ocular Oncology

and Pathology
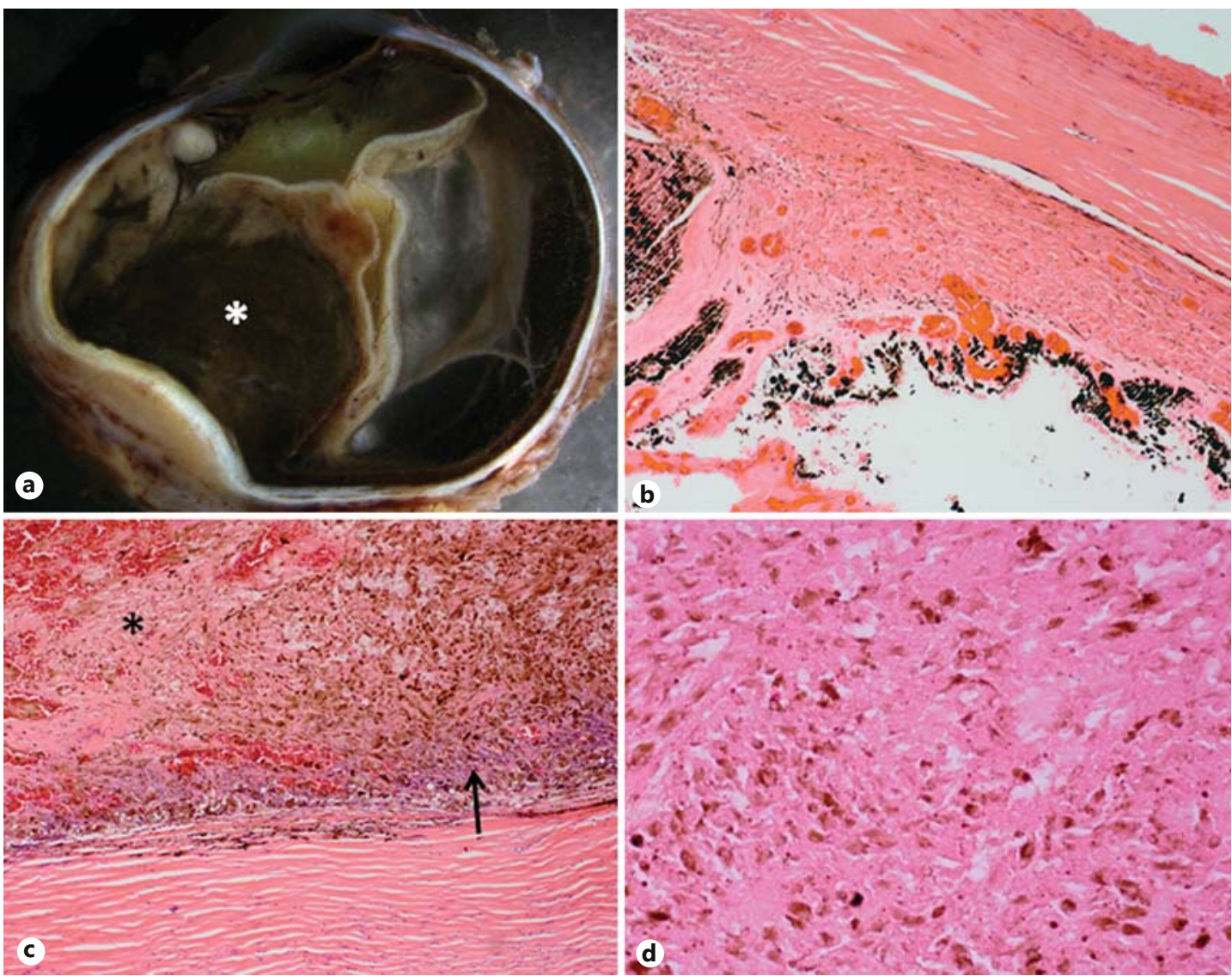

Fig. 2. Case 2. a Gross appearance of the enucleated eye with a bilobed melanoma. The posterior aspect of the tumor is pigmented $\left({ }^{*}\right)$, whereas the anterior aspect is amelanotic. $\mathbf{b}$ The ciliary body exhibits ischemic necrosis including pale eosinophilic staining, dilated vascular channels, and clumping of the iris pigment epithelium. c Nearly $100 \%$ of the tumor is necrotic $\left({ }^{*}\right)$, although a thin rim of occasional intact tumor cells (arrow) is present near the sclera. d The necrotic tumor consists of ghost melanoma cells, some of which contain melanin pigment in their cytoplasm. b, c HE. $\times 25$. d HE. $\times 100$.

with a zone of pigmented macrophages. All cases contained ghost melanoma cells, and in most of the cases erythrocytes were admixed with the tumor. All of these cases exhibited the concomitant findings of ischemic necrosis in both the iris and ciliary body. In 5 cases, there was also necrosis of the retina, while the remaining case showed mild ischemia to the retina. We did not find vasculitis or marked inflammation of the tumor in any of the cases, although there was inflammation including perivasculitis in the episclera. We did not find thrombi in any long posterior ciliary artery, short posterior ciliary artery or the major circle of the iris in any of the cases.

\section{Discussion}

The 6 patients with spontaneously necrotic uveal melanomas in our study exhibited similar clinical presentations to those in previous studies [1-6]. All of them presented with pain as a common clinical finding in addition to acute changes in vision, inflammation of the sclera and episclera [7], and raised intraocular pressure. All tumors exhibited $\geq 90 \%$ tumor 


\section{Ocular Oncology \\ and Pathology}

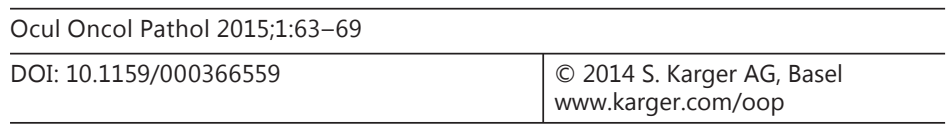

Thareja et al.: Spontaneous Necrosis of Choroidal Melanoma

necrosis, and all of our 6 cases exhibited marked ischemic necrosis of the iris and ciliary body with 5 of 6 cases showing marked retinal ischemia.

The mechanisms of necrosis of melanomas and ocular structures as well as orbital inflammation as a consequence of necrosis are not fully understood. Brannan et al. [4] discussed 2 potential mechanisms of infarction with associated ocular necrosis. In the first mechanism, the tumor mass in the posterior segment raises the intraocular pressure, leading to an acute angle closure, vascular compromise, and ischemia of the ocular contents. In the second mechanism, tumor necrosis, triggered by an unknown mechanism, leads to the release of cytotoxic products. These irritants can lead to vasculitis of intraocular vessels and consequently to infarction, swelling, and cellulitis of ocular and extraocular tissues. Vasculitis in the anterior ciliary arteries, central retinal artery, and choriocapillaries supported Brannan et al.'s hypothesis. Vasculitis provides a potential explanation for the infarction of both the anterior and posterior choroid, which typically avoid vascular compromise due to vessel anastomoses [4].

Evidence also exists for the aggravation of the inflammatory response from tumor necrosis due to cytotoxic agents released from dying melanocytes. In a study by Reese et al. [8], 2 types of necroses were observed in 22 cases of necrotic uveal melanoma. In the cellular necrosis cases, plasma cells and lymphocytes were seen in the inflammatory infiltrate in the eye along with dispersed free melanin in the tumor, retina, vitreous, and choroid. In the coagulative (ischemic) necrosis cases, there was evidence of cellular necrosis and an additional vascular insult. These findings supported Reese et al.'s hypothesis that chronic inflammatory events occur alongside necrosis and that sudden infarction then leads to acute pain. These cases also had clear zones of lymphocytes, demarcating the viable and necrotic tissue. The tumor cells were postulated to provoke a T-cell and B-cell response, with the T cells injuring the vessel endothelium and leading to thrombosis, infarction, and necrosis [8]. In both Brannan et al.'s [4] and Reese et al.'s [8] work, the idea was that either inflammation or secondary glaucoma occurred first, followed by ischemic necrosis of the tumor.

The potential for vascular compromise occurring first and initiating necrosis has not been given much attention in this process. Wolter [9] discussed vascular occlusion initiating necrotic events in choroidal melanomas and ocular tissues. Some uveal melanomas may be predisposed to necrosis due to fast growth, which is not sufficiently supported by their established vascular supply. In Wolter's case, the iris, ciliary body, and choroidal necrosis were initiated by vascular occlusion of the long posterior ciliary artery, which was due to giant cell arteritis. While blood was present in the vessel lumen in the area of inflammation around the necrosis, there was only exudate in the lumen in the sclera and choroid at the tumor location [9].

Patterns of tumor infarction can inform us of the source of the vascular insult. Watershed infarction of a choroidal tumor requires vascular compromise from both the anterior and posterior circulation of the eye (fig. 3). We believe that complete infarction of the tumors that were examined in our study likely progressed from watershed infarctions that spread to include the entire tumors as ischemia continued. These infarction processes can be better understood by reviewing the vascular supply to the eye. The short and long posterior ciliary arteries, which are branches of the ophthalmic artery, are responsible for supplying the choroid as well as the iris and ciliary body, respectively. The latter occurs via anastomosis at the ciliary plexus with the anterior ciliary artery. The anterior ciliary artery branches from the lacrimal artery, which is a continuation of the ophthalmic artery after the long posterior ciliary has separated, and forms a vascular network in the iris and ciliary body.

All of the tumors in our cases were centered around the equatorial choroid, with 2 extending into the ciliary body. The senior author (H.E.G.) has occasionally observed wedgeshaped areas of spontaneous infarctions in the centers of choroidal melanomas that straddle 
Ocular Oncology

and Pathology

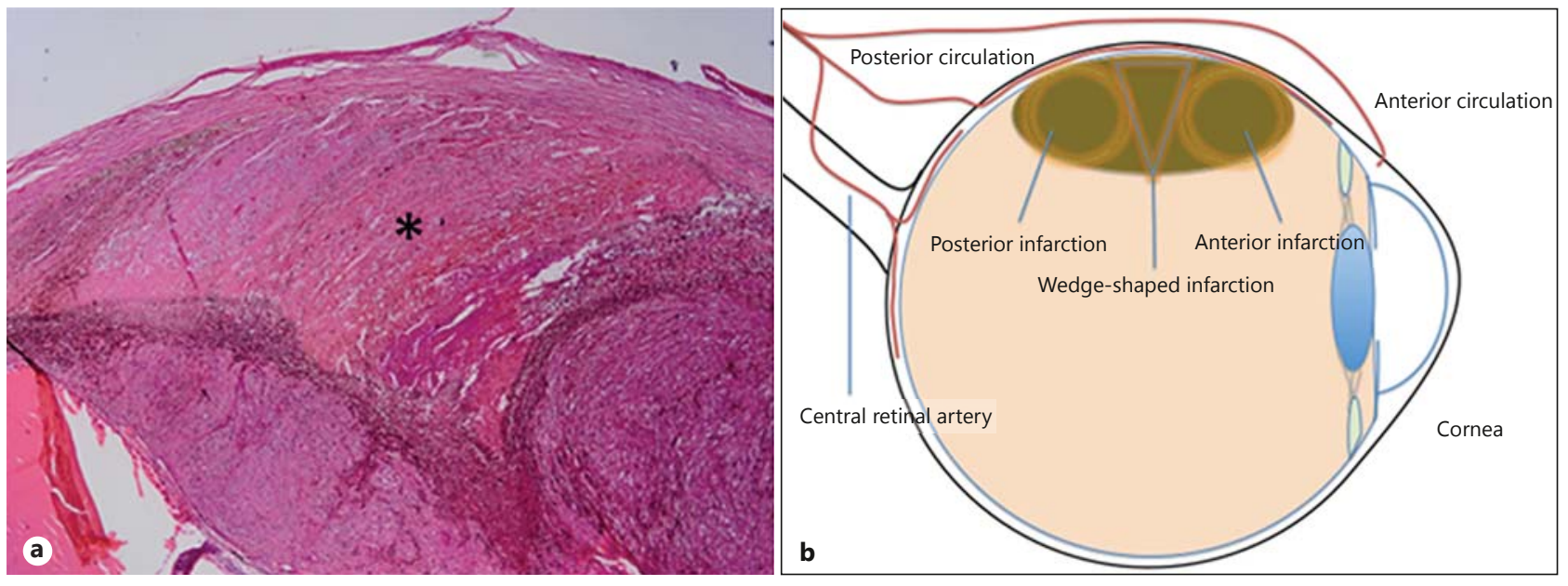

Fig. 3. a Example of a wedge-shaped infarct $\left({ }^{*}\right)$ in a case of choroidal melanoma. b Diagram of the vascular supply to an eye containing a choroidal melanoma. We hypothesize that hypoxia occurs in the center of the tumor that may result in a wedge-shaped area of infarction followed by anterior and posterior infarction. This ischemia may result in retinal detachment, secondary glaucoma, and necrosis-related inflammation.

the equator, which supports the concept of spontaneous watershed infarction (fig. 3). One of our cases (case 2) exhibited a wedge-shaped infarct in a lateral aspect of the tumor. In the remaining cases, the lateral aspect of the tumors was either not sectioned or completely necrotic. If tumor cells were intact in any of our cases, this occurred at the periphery of the tumor, thus supporting the concept of watershed ischemic necrosis in the center of the tumor with outward expansion of ischemic necrosis. We believe that this is due to hypoxia within the center of the tumor from the tumor outgrowing its blood supply, leading to ischemic necrosis with a release of cytokines, followed by some inflammation in the surrounding tissues including perivascular inflammation in the episclera. This is supported by a lack of thrombi in the long posterior ciliary arteries, short posterior ciliary arteries, and the major circle of the iris. There may be associated necrosis of the iris, ciliary body, and retina due to inflammation and ischemia. We hypothesize that the center of the tumor outgrows its blood supply in the watershed area, and ischemic necrosis of the tumor results in the release of cytokines and tumor swelling with further necrosis, anterior displacement of the lens iris diaphragm, and secondary glaucoma. This resultant increased intraocular pressure potentiates further tumor necrosis with associated necrosis of the iris and ciliary body.

In summary, virtually complete tumor necrosis can occur spontaneously in some cases of choroidal melanoma. We believe that this the result of hypoxia in the center of the tumor due to the tumor outgrowing its blood supply centrally in the watershed area, and later complete tumor necrosis. Inflammation and angle-closure glaucoma appear to occur as secondary changes. Raised intraocular pressure potentiates this process. These patients present with pain, inflammatory signs and symptoms, and usually require enucleation.

\section{Acknowledgment}

This work was supported in part by an unrestricted departmental grant from Research to Prevent Blindness, Inc., and NIH NEIP30EY06360. 


\section{References}

1 Palamar M, Thangappan A, Shields CL, Ehya H, Shields JA: Necrotic choroidal melanoma with scleritis and choroidal effusion. Cornea 2009;28:354-356.

-2 Bujara K: Necrotic malignant melanomas of the choroid and ciliary body. A clinicopathological and statistical study. Graefes Arch Clin Exp Ophthalmol 1982;219:40-43.

-3 Moshari A, Cheeseman EW, McLean IW: Totally necrotic choroidal and ciliary body melanomas: associations with prognosis, episcleritis, and scleritis. Am J Ophthalmol Feb 2001;131:232-236.

4 Brannan S, Browne B, Clark BJ: Massive infarction of ocular tissues complicating a necrotic uveal melanoma. Eye (Lond) 1998;12:324-325.

5 Bhagat S, Ramaesh K, Wharton SB, Dhillon B: Spontaneous acute scleritis and scleral necrosis in choroidal malignant melanoma. Eye (Lond) 1999;793-795.

6 Tabassian A, Zuravieff JJ: Necrotic choroidal melanoma with orbital inflammation. Arch Ophthalmol Dec 1995; 113:1576-1577.

-7 Fraser DJ Jr, Font RL: Ocular inflammation and hemorrhage as initial manifestations of uveal malignant melanoma. Incidence and prognosis. Arch Ophthalmol 1979;97:1311-1314.

$>8$ Reese AB, Archila EA, Jones IS, Cooper WC: Necrosis of malignant melanoma of the choroid. Am J Ophthalmol 1970;69:91-104.

-9 Wolter JR: Necrosis of choroidal melanoma: in ciliary artery involvement with temporal arteritis. $\mathrm{Br} \mathrm{J}$ Ophthalmol 1989;73:216-219. 